\title{
Pontas hidráulicas, adjuvante e horários de aplicação de fomesafen no controle de picão-preto ${ }^{1}$
}

\author{
Nozzles hydraulic, adjunctive and fomesafen application time in controlling \\ beggar's tick
}

\begin{abstract}
João de Deus Godinho Junior²; José Márcio de Sousa Júnior³; Guilherme Andrade Gontijo; Renato Adriane Alves Ruas ${ }^{5}$
\end{abstract}

Resumo - A aplicação de defensivos agrícolas é afetada por diversos fatores, dentre eles, destacase o momento da aplicação, que pode variar de acordo com o alvo, o produto aplicado e equipamento utilizado. Objetivou-se avaliar pontas hidráulicas, adjuvante e horários de aplicação de fomesafen no controle de picão-preto. Os tratamentos foram arranjados em esquema fatorial $4 \times 2 \times 2$, com o primeiro fator constituído de quatro pontas (API-11002; jato leque simples-JLS, AD/D-11002; jato leque duplo-JLD, AVI TWIN-11002; jato leque duplo com indução de arJLDIA, e APM-09502; jato leque simples de impacto-JLSIP). O segundo de dois horários de aplicação (3 e 16 horas) e o terceiro de duas misturas em tanque (com e sem adição de adjuvante espalhante adesivo não iônico). Os tratamentos foram avaliados sete, 14 e 21 dias após à aplicação, através do número de plantas vivas, altura das plantas, análise visual e aos 21 dias foi feita matéria seca da parte aérea. A aplicação às 16 horas proporcionou maior controle de todas as características avaliadas, a ponta JLSIP se destacou causando maior redução de matéria seca e de plantas vivas e a adição de adjuvante potencializou o controle da aplicação às 16 horas. O horário de aplicação influencia a ação do herbicida fomesafen, sendo que, sua aplicação às 16 horas proporciona maior controle de B. pilosa em relação à realizada às 3 horas. A ponta JLSIP aumenta a ação de fomesafen e a adição de adjuvante potencializa o efeito desta ponta e da aplicação realizada às 16 horas.

Palavras-chaves: Bidens pilosa; condições climáticas; horário de aplicação; inibidor PROTOX

Abstract - The application of pesticides is affected by several factors. Among them, the time of application stands out, which can vary according to the target, the applied product and equipment used. This study aimed to evaluate hydraulic nozzles, adjuvant and fomesafen application time in controlling beggar's tick. The treatments were arranged in a factorial $4 \times 2 \times 2$, with the first factor consisting in four nozzles (API-11002; even (E) flat-fan, AD/D-11002; twin flat spray, AVI TWIN-11002; air induction (AI) twin flat spray-TQ, and APM-09502; Flood jet spray). The second with two application times ( 3 and 16 hours) and the third in two tank mixtures (with and without the addition of nonionic adhesive spreader adjuvant). The treatments were evaluated at 7,14 and 21 days after the application, through the number of live plants, plant height, visual analysis and at 21 days the dry matter of the aerial part was made. The application at 16 hours provided better

\footnotetext{
${ }^{1}$ Recebido para publicação em 07/12/2015 e aceito em 13/02/2016.

${ }^{2}$ Engenheiro Agrônomo, Mestrando, Instituto de Ciências Agrárias, Universidade Federal de Viçosa, UFV, Rodovia MG-230, Km 7 - Zona Rural, Rio Paranaíba - MG, 38810-000, Brasil. E-mail: joao.ufv.agro@ gmail.com.

${ }^{3}$ Engenheiro Agrônomo, Mestrando, UFV, Rio Paranaíba/MG, Brasil.

${ }^{4}$ Engenheiro Agrônomo, São Gotardo/MG, Brasil.

${ }^{5}$ Engenheiro Agrônomo, Professor Adjunto IV, UFV, Rio Paranaíba/MG, Brasil.
} 
control of all traits, the JLSIP nozzle stood out causing further reduction of dry matter and of living plants, and the addition of adjuvant potentiated the application control at 16 hours. The application time influences the action of fomesafen, and that its application at 16 hours provides greater control of $B$. pilosa compared to that performed at 3. The JLSIP nozzle increases fomesafen action, also the addition of adjuvant potentiates the effect of this nozzle and the application held at 16 hours.

Keywords: Bidens pilosa; weather conditions; application time; PROTOX inhibitor

\section{Introdução}

Os herbicidas são amplamente utilizados na agricultura, tanto no sistema de plantio convencional quanto no sistema de plantio direto na palha, para controle de plantas daninhas e também na dessecação de plantas utilizadas como cobertura vegetal. Uma das principais plantas daninhas que interfere na produtividade das lavouras do Brasil é o picãopreto (Bidens pilosa) (Santos e Cury, 2011). Condições tropicais e o fato de ser uma planta de desenvolvimento rápido, com alta produção de sementes, garantem infestações durante todo ano nas lavouras, elevando, assim, seu potencial de interferência sobre culturas.

Um dos herbicidas utilizados no controle de B. pilosa e que propicia bom controle, quando aplicado nas doses recomendadas, é o fomesafen. Este herbicida inibe a enzima protoporfirinogênio oxidase (PROTOX) durante a biossíntese de clorofila, sua ação herbicida ocorre na presença de luz (fotossíntese) e oxigênio, ocasionando a morte celular através da oxidação dos lipídeos das membranas celulares (Grimm, 1999; Wakabayashi e Böger, 1999). Entretanto, para que isso ocorra, é fundamental que o ingrediente ativo do herbicida atinja o alvo. Para isso, é indispensável conhecer tanto os aspectos de distribuição da calda gerados pelos equipamentos como, também, as condições climáticas do ambiente que irão interagir com a dinâmica das gotas de pulverização, as quais são responsáveis pelo transporte do produto até o alvo. As condições climáticas variam conforme o local e no decorrer do dia e não podem ser controladas, ao contrário do modelo de ponta e o adjuvante utilizados, porém, essas condições do ambiente afetam diretamente a distribuição e a eficácia do produto aplicado (Ferreira et al., 1998; Pitelli et al., 2011).

A redução da umidade e/ou elevação da temperatura, diminui o tempo de vida útil das gotas aplicadas, reduzindo ou até interrompendo a absorção do produto aplicado (Rodrigues et al., 2011). Já a ocorrência de orvalho pode reduzir a eficácia das pulverizações, principalmente naquelas realizadas com menores doses e com maiores volumes de calda, pois favorece a diluição e o escorrimento da calda aplicada (Roman et al., 2004). Para tentar compensar a interferência desses fatores ambientais nas aplicações, novas linhas de pesquisa vêm projetando equipamentos que se adequam às variações meteorológicas do ambiente (Alvarenga et al., 2013; Stanislavski et al., 2014). No entanto, para isso, é fundamental conhecer os efeitos em campo dessas variações na qualidade da aplicação.

Existem diversos recursos tecnológicos visando o aprimoramento dos controles das plantas daninhas. Dentre esses recursos, podemos citar os diferentes modelos de pontas hidráulicas disponíveis. Um exemplo, são as pontas acrescidas de indução de ar, as quais vem sendo testadas a fim de aprimorar a qualidade das aplicações. Essas pontas produzem gotas com menor massa, que são menos susceptíveis ao ricocheteio e ao escorrimento, por outro lado, o seu tamanho extremamente grosso afeta negativamente a cobertura e distribuição do produto aplicado (Grigolli et al., 2011).

Juntamente com as pontas, os adjuvantes constituem importante ferramenta da tecnologia de aplicação. São produtos químicos que promovem alterações na calda, atenuando os adversos efeitos ambientais e morfofisiológicos da planta, elevando a eficácia do controle. Eles tornam a calda menos susceptível às variações 
de umidade relativa do ar e temperatura ambiente (Nascimento et al., 2012; Vilela et al., 2013). Entretanto, seu efeito no espectro de gotas pode variar de acordo com a ponta hidráulica e o momento de aplicação (Gandolfo et al., 2013). Entre os adjuvantes, temos os surfactantes que agem diminuindo a tensão superficial das gotas pulverizadas, contribuindo para a formação de filmes líquidos sobre a superfície foliar (Montório et al., 2005), aumentando assim a superfície de contato e consequentemente a absorção do herbicida aplicado.

Portanto, objetivou-se avaliar pontas hidráulicas, adjuvante e horários de aplicação de fomesafen no controle de picão-preto.

\section{Material e Métodos}

O experimento foi realizado em campo experimental na Universidade Federal de Viçosa Campus Rio Paranaíba (19²'50.47"S 46¹3'54.64"W), Latossolo Vermelho-Amarelo distroférrico típico de textura argilosa, e clima tipo Cwb segundo Köppen.

Em delineamento inteiramente casualizado (DIC), com quatro repetições. Os tratamentos foram arranjados em esquema fatorial $4 \times 2 \times 2$, com 0 primeiro fator correspondente a quatro pontas hidráulicas, API-11002 jato leque simples-JLS, AD/D11002, jato leque duplo-JLD, AVI TWIN11002 jato leque duplo com indução de arJLDIA, e APM-09502 jato leque simples de impacto-JLSIP, o segundo a dois horários de aplicação, três e 16 horas e o terceiro a duas misturas em tanque (com e sem adição de adjuvante espalhante adesivo não iônico, marca comercial Haiten.

A área experimental $\left(480 \mathrm{~m}^{2}\right)$ foi preparada com uma gradagem pesada seguida de enxada rotativa, onde foram estabelecidas 70 linhas de semeadura. Em cada linha, com três metros de comprimento e espaçadas $0,5 \mathrm{~m}$ das demais, foram distribuídas em torno de 15 sementes de $B$. pilosa a cada $10 \mathrm{~cm}$. Após a germinação das sementes, foi realizado desbaste, deixando-se cerca de três plantas a cada $10 \mathrm{~cm}$.

A aplicação de fomesafen (Flex, $250 \mathrm{~g}$ $\mathrm{L}^{-1}$ i.a., SL, Syngenta) foi feita na dose de $225 \mathrm{~g}$ ha $^{-1}$ i.a., cinco semanas após a emergência das sementes (35 dias após a emergência). Utilizouse pulverizador costal pressurizado com $\mathrm{CO}_{2}$, na pressão de trabalho de $300 \mathrm{kPa}$ e altura de aplicação de 0,5 m. No momento da aplicação, protegeu-se as linhas adjacentes com estrutura de lona, construída com o objetivo de evitar os efeitos da deriva sobre os demais tratamentos.

As condições climáticas no momento da aplicação foram: umidade relativa do ar de 90,5 e $87,5 \%$, temperatura ambiente de 19 e $22^{\circ} \mathrm{C}$, para à aplicação das três e 16 horas respectivamente. A devido a utilização da proteção contra deriva, a velocidade do vento foi zero em ambas as aplicações, e na aplicação realizada as três horas havia presença de orvalho sobre as folhas.

As avaliações foram feitas aos 7, 14 e 21 dias após à aplicação de fomesafen, através de contagem do número de plantas vivas por linha, medição da altura das plantas e análise visual de controle em relação às quatro parcelas onde não houve aplicação do herbicida, as testemunhas. Para isso, foi empregada a escala proposta pela Asociación Latinoamericana de Malezas (ALAM, 1974), que indica notas variando de $0 \%$ (nenhum controle) até $100 \%$ (controle total).

Aos 21 dias após à aplicação, as plantas foram cortadas rente à superfície do solo. Em seguida, colocadas para secar em estufa a $70{ }^{\circ} \mathrm{C}$ durante $72 \mathrm{~h}$ e a matéria seca (MS) determinada.

Os dados foram submetidos a teste de normalidade e, se necessário, análise de dados não paramétricos pelo teste de Kruskal-Wallis.

\section{Resultados e Discussão}

O teste de normalidade não foi positivo, os dados não tiveram distribuição normal e foram então analisados pelo teste não paramétrico de Kruskal-Wallis.

A porcentagem de controle visual foi significativa apenas para o horário em que foi 
realizada a aplicação, na avaliação realizada aos sete e 14 dias após a aplicação do fomesafen, onde, a aplicação às 16 horas proporcionou maior controle (Tabela 1). Talvez, um dos fatores que tenha contribuindo para esses resultados seja, à presença de orvalho sobre as folhas na aplicação realizada às três horas. A presença de orvalho pode favorecer a dispersão e escorrimento do produto aplicado sobre a superfície foliar, diminuindo assim seu efeito (Roman et al., 2004). Discordando de Lewis et al. (2013), que observaram um aumento da eficácia do herbicida aminocyclopyrachlor quando aplicado na presença de orvalho.

Tabela 1. Porcentagem de controle de B. pilosa aos 7, 14 e 21 dias após à aplicação (DAA) de fomesafen em dois horários: 3 e 16 horas.

\begin{tabular}{lccc}
\hline \multirow{2}{*}{$\begin{array}{c}\text { Horário de } \\
\text { aplicação }\end{array}$} & \multicolumn{3}{c}{ Porcentagem de controle visual } \\
\cline { 2 - 4 } & 7 & 14 & 21 \\
\hline 3:00 & $31 \mathrm{~b}$ & $43 \mathrm{~b}$ & $50 \mathrm{a}$ \\
16:00 & $59 \mathrm{a}$ & $61 \mathrm{a}$ & $59 \mathrm{a}$ \\
\hline *As médias seguidas por mesmas letras, minúsculas nas colunas, não \\
diferem entre si pelo teste de Kruskal-Wallis (P>0,05). CV (\%): 30 (sete \\
dias); 34 (14 e 21 dias). Diferença crítica: 9.
\end{tabular}

A média das repetições de cada tratamento avaliando-se o percentual de plantas mortas, foi significativo para horário de aplicação e para a interação entre modelo de ponta e horário de aplicação (Tabela 2). Sendo que, a aplicação realizada às 16 horas gerou maior número de plantas mortas em relação a aplicação realizada as três horas. Desdobrando a interação para pontas dentro de cada horário, houve diferença estatística significativa apenas aos sete dias após a aplicação, e somente na aplicação das 16 horas, onde, as pontas JLSIP e JLD proporcionaram maior controle. Pontas do modelo JLSIP produzem gotas grossas que são menos susceptíveis à deriva e ao mesmo tempo uma boa deposição, sendo assim, indicadas para situações climáticas mais adversas e/ou quando se deseje diminuir ao máximo o potencial risco de deriva (Souza, 2012).

Agora, em relação ao horário de aplicação dentro de cada modelo de ponta, houve significância nas avaliações realizadas 14 e 21 dias após a aplicação e apenas no modelo JLSIP. Onde, a aplicação realizada às 16 horas proporcionou quase cinco vezes mais plantas mortas em comparação a aplicação feita as três horas (Tabela 2). Esses resultados podem ser atribuídos, a ausência de luz durante a aplicação realizada as três horas. Pois, a não ocorrência de fotossíntese ao longo e ou logo após à aplicação do herbicida fomesafen, restringem o seu efeito no controle de B. pilosa (Ferreira et al., 1998). Concordando com Cieslik (2014), que propõem que o maior controle de fomesafen em plantas daninhas de folha larga é alcançado quando o herbicida é aplicado em maiores temperaturas e elevados níveis de irradiância.

Em relação a porcentagem de redução da altura, houve significância 14 e 21 dias após a aplicação e apenas para o horário de aplicação, sendo que, a aplicação às 16 horas proporcionou maior redução da altura (Tabela 3 ). Sendo que, a redução da altura aumentou até os 14 dias após a aplicação de fomesafen e depois tendeu a diminuir (Figura 1). Isso demonstra uma redução gradual do efeito do herbicida absorvido pelas plantas.

Por fim, a avaliação da matéria seca foi significativa para modelo de ponta e horário de aplicação, onde, os modelos JLS e JLSIP se destacaram e proporcionaram as maiores reduções de matéria seca. Em relação ao momento da aplicação, a aplicação das 16 horas reduziu em $77 \%$ a matéria seca e a aplicação das três horas 70\% (Tabela 4). Concordando com Stopps (2013), que sugere que herbicidas de aplicação em pós emergência são mais eficazes quando aplicadas durante o dia. Testando-se três herbicidas, entre eles o acifluorfen, de modo de ação semelhante ao fomesafen, em cinco horários de aplicação, Gazziero e Fleck (1980) não encontraram diferença significativa no controle de $B$. pilosa. Porém, observaram tendência de menor matéria seca nas plantas de B. pilosa pulverizadas na parte da tarde, e relatam que o aumento da temperatura diminuiu a matéria seca das plantas controladas com o herbicida acifluorfen. 
Tabela 2. Porcentagem de plantas de B. pilosa mortas aos 7, 14 e 21 dias após à aplicação de fomesafen em dois horários: 3 e 16 horas, com quatro modelos de pontas: jato leque simples-JLS; jato leque duplo-JLD; jato leque duplo com indução de ar-JLDIA; jato leque simples de impactoJLSIP.

\begin{tabular}{|c|c|c|c|c|c|c|}
\hline \multirow{3}{*}{$\begin{array}{l}\text { Dias após à } \\
\text { aplicação }\end{array}$} & \multirow{3}{*}{$\begin{array}{l}\text { Horário de } \\
\text { aplicação }\end{array}$} & \multicolumn{5}{|c|}{ Porcentagem de plantas mortas* } \\
\hline & & \multicolumn{4}{|c|}{ Ponta hidráulica } & \multirow[t]{2}{*}{ Média dos horários } \\
\hline & & JLS & JLD & JLDIA & JLSIP & \\
\hline \multirow{2}{*}{7} & $3: 00$ & $15 \mathrm{Aa}$ & $8 \mathrm{Aa}$ & $8 \mathrm{Aa}$ & $10 \mathrm{Aa}$ & $10 \mathrm{~b}$ \\
\hline & $16: 00$ & $13 \mathrm{Ba}$ & $36 \mathrm{ABa}$ & $10 \mathrm{Ba}$ & $50 \mathrm{Aa}$ & $27 \mathrm{a}$ \\
\hline \multirow{2}{*}{14} & $3: 00$ & $16 \mathrm{Aa}$ & $14 \mathrm{Aa}$ & $15 \mathrm{Aa}$ & $8 \mathrm{Ab}$ & $13 \mathrm{~b}$ \\
\hline & 16:00 & $17 \mathrm{Aa}$ & $24 \mathrm{Aa}$ & $26 \mathrm{Aa}$ & $52 \mathrm{Aa}$ & $30 \mathrm{a}$ \\
\hline \multirow{2}{*}{21} & 3:00 & $18 \mathrm{Aa}$ & $12 \mathrm{Aa}$ & $13 \mathrm{Aa}$ & $13 \mathrm{Ab}$ & $14 \mathrm{~b}$ \\
\hline & $16: 00$ & $15 \mathrm{Aa}$ & $36 \mathrm{Aa}$ & $33 \mathrm{Aa}$ & $52 \mathrm{Aa}$ & $34 \mathrm{a}$ \\
\hline
\end{tabular}

*As médias para cada período de avaliação (sete, 14 e 21 dias), seguidas por mesmas letras, maiúsculas nas linhas e minúsculas nas colunas, não diferem entre si pelo teste de Kruskal-Wallis (P>0,05). CV (\%): 62 (sete dias); 77 (14 dias); 69 (21 dias). Diferença critica: 29 para a interação ponta x horário; para o horário: 9.

Tabela 3. Porcentagem de redução do crescimento de B. pilosa aos 7, 14 e 21 dias após a aplicação (DAA) de fomesafen em dois horários: 3 e 16 horas.

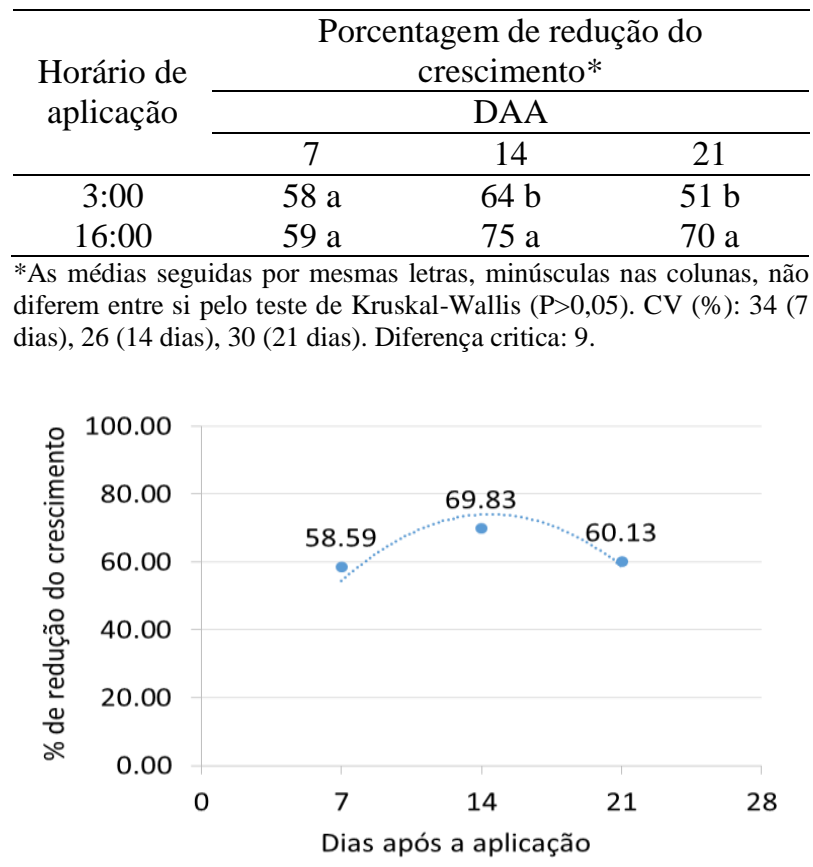

Figura 1. Redução do crescimento de B. pilosa aos 7, 14 e 21 dias após à aplicação de fomesafen.

Comparando-se os dois horários de aplicação, ambos com a adição de adjuvante, houve uma diferença significância na redução da matéria seca. Onde, a aplicação realizada as 16 horas proporcionou maior controle em relação a aplicação feita as três horas (Tabela 4).
Além disso, quando adicionando à calda, no caso da aplicação realizada as 16 horas e com a ponta JLSIP, a adição de adjuvante diminuiu em $40 \%$ a matéria seca das plantas. Controle significativamente maior em relação a mesma aplicação sem o acréscimo de adjuvante.

$A$ adição de adjuvante surfactante na mistura de tanque reduz a tensão superficial das gotas pulverizadas, e contribui assim para a absorção do ingrediente ativo aplicado (Maciel et al., 2011; Oliveira e Antuniassi, 2012). Ademais, a entrada do herbicida via estômatos só é possível com essa redução da tensão superficial da calda aplicada (Oliveira e Bacarin, 2011; Inacio e Monquero, 2013). O mesmo adjuvante aqui avaliado, quando comparado a outros surfactantes não siliconados, se destaca proporcionando os maiores índices de eficácia e de redução da tensão superficial (Montório et al., 2005).

De modo geral, os melhores resultados no controle de $B$. pilosa foram alcançados aplicando-se o herbicida fomesafen as 16 horas, utilizando a ponta JLSIP e com a adição de adjuvante espalhante adesivo não iônico na mistura de tanque. Em contrapartida, o menor controle ocorreu quando se realizou a aplicação as três horas, não havendo nesse horário de aplicação significância entre os modelos de pontas hidráulicas e a adição ou não de adjuvante na mistura de tanque. 
Tabela 4. Porcentagem de redução da matéria seca da parte área de $B$. pilosa aos 21 dias após à aplicação de fomesafen, em dois horários de aplicação: três e 16 horas e com quatro modelos de pontas: jato leque simples-JLS; jato leque duplo-JLD; jato leque duplo com indução de ar-JLDIA; jato leque simples de impacto-JLSIP.

\begin{tabular}{|c|c|c|c|c|c|c|c|}
\hline \multirow{3}{*}{$\begin{array}{l}\text { Horário de } \\
\text { aplicação }\end{array}$} & \multicolumn{7}{|c|}{ Porcentagem de redução de matéria seca* } \\
\hline & \multicolumn{4}{|c|}{ Ponta hidráulica (média dos horários) } & \multirow{2}{*}{$\begin{array}{l}\text { Média dos } \\
\text { horários }\end{array}$} & \multicolumn{2}{|c|}{ Adjuvante } \\
\hline & JLS & JLD & JLDIA & JLSIP & & Com & Sem \\
\hline $3: 00$ & & & & & $70 \mathrm{~b}$ & $67 \mathrm{Ab}$ & $72 \mathrm{Aa}$ \\
\hline 16:00 & $81 \mathrm{~A}$ & $72 \mathrm{~B}$ & $64 \mathrm{~B}$ & $76 \mathrm{AB}$ & $77 \mathrm{a}$ & $81 \mathrm{Aa}$ & $73 \mathrm{Aa}$ \\
\hline
\end{tabular}

*As médias seguidas por mesmas letras, maiúsculas nas linhas e minúsculas nas colunas, não diferem entre si pelo teste de Kruskal-Wallis $(\mathrm{P}>0,05)$. CV (\%): 9. Diferença critica: 17 (ponta e adjuvante) e 9 (horário).

\section{Conclusões}

O horário de aplicação influencia a ação do herbicida fomesafen, sendo que, sua aplicação às 16 horas proporciona maior controle de $B$. pilosa em relação à realizada às três horas.

A ponta hidráulica jato leque simples de impacto aumenta a ação de fomesafen. A adição de adjuvante espalhante adesivo não iônico a mistura de tanque, potencializa o efeito da ponta hidráulica jato leque simples de impacto e da aplicação realizada às 16 horas.

\section{Agradecimentos}

Ao programa $\mathrm{CNPq} / \mathrm{PIBIC}$ pelo suporte financeiro dado a essa pesquisa.

\section{Referências}

Alvarenga, C.B.; Teixeira, M.M.; Zolnier, S.; Sasaki, R.S.; Rinaldi, P.C.N. Controle automático do espectro de gotas de pulverizador hidropneumático em função do déficit de pressão de vapor d'água no ar. Pesquisa Agropecuária Tropical, v.43, n.1, p.26-33, 2013.

\section{ASOCIACIÓN LATINOAMERICANA DE} MALEZAS - ALAM. Recomendaciones sobre unificación de los sistemas de evaluación en ensayos de control de malezas. ALAM, v.1, n.1, p.35-38, 1974.

Cieslik, L.F.; Vidal, R.A.; Trezzi, M.M. Fomesafen toxicity to bean plants as a function of the time of application and herbicide dose. Acta Scientiarum, v.36, n.3, p.329-334, 2014
Lewis, D.F.; Jeffries, M.D.; Strek, H.J.; Richardson, R.J.; Yelverton, F.H. effect of ambient moisture on aminocyclopyrachlor efficacy. Weed Technology, v.27, n.2, p.317$322,2013$.

Ferreira, M.C.; Machado Neto, J.G.; Matuo, T. Redução da dose e do volume de calda nas aplicações noturnas de herbicidas em pósemergência na cultura de soja. Planta Daninha, v.16, n.1, p.25-36, 1998.

Gandolfo, M.A.; Chechetto, R.G.; Carvalho, F.K.; Gandolfo, U.D.; Moraes, E.D. Influência de pontas de pulverização e adjuvantes na deriva em caldas com glyphosate. Revista Ciência Agronômica, v.44, n.3, p.474-480, 2013.

Gazziero, D.L.P.; Fleck, N.G. Efeitos de três herbicidas pós-emergentes aplicados em diferentes horas do dia sobre ervas daninhas e plantas de soja [Glycine $\max$ (L.) Merrill]. Planta Daninha, v.3, n.1, p.23-29, 1980.

Grigolli, J.F.J.; Pereira, F.C.M.; Peñaherrera, L.C.; Santos, E.A.; Ferreira, M.C.F. Controle de Euphorbia heterophylla com mesotrione e óleos para diferentes pontas de pulverização. Revista Brasileira de Herbicidas, v.10, p.266-276, 2011.

Grimm, B. The metabolic pathway of tetrapyrrole biosynthesis. Springer-Verlag, Berlin Heidelberg, Germany, p.213-244, 1999.

Inacio, E.M.; Monquero, P.A. Controle químico e caracterização da superfície foliar de Conyza bonariensis e $C$. canadensis (Asteraceae). Revista Brasileira de Herbicidas, v.12, n.3, p.220-231, 2013. 
Maciel, C.D.G.; Oliveira Neto, A.M.; Guerra, N.; Justiniano, W. Eficiência e qualidade da aplicação de misturas em tanque com adjuvantes na dessecação de corda-de-viola. Engenharia Agrícola, v.31, n.4, p.704-715, 2011.

Montório, G.A.; Velini, E.D.; Maciel, C.D.G.; Montório, T. Eficiência dos surfactantes de uso agrícola na redução da tensão superficial. Revista Brasileira de Herbicidas, v.4, n.2, p.822, 2005.

Nascimento, A.B.; Oliveira, G.M.; Balan, M.G.; Higashibara, L.R.; Abi Saab, O.J.G. Deposição de glifosato e utilização de adjuvante para diferentes pontas de pulverização e horário de aplicação. Pesquisa Aplicada \& Agrotecnologia, v.5, n.2, p.105-116, 2012.

Oliveira JR., R.S.; Bacarin, M.A. Absorção e translocação de herbicidas. Biologia e Manejo de Plantas Daninhas. 1ed.Curitiba, PR: Omnipax Editora Ltda., 2011, v.1, p.215-242, 2011.

Oliveira, R.B.; Antuniassi, U.R. Caracterização física e química e potencial de deriva de caldas contendo surfactantes em pulverizações agrícolas. Energia na Agricultura, v.27, n.1, p.138-149, 2012.

Pitelli, R.A.; Bisigatto, A.T.; Kawaguchi, I.; Pitelli, R.L.C.M. Doses e horário de aplicação do diquat no controle de Eichhornia crassipes.

Planta Daninha, v.29, n.2, p.269-277, 2011.

Rodrigues, E.B.; Abi Saab, O.J.G.; Gandolfo, M.A. Cana-de-açúcar: avaliação da taxa de aplicação e deposição do herbicida glifosato. Revista Brasileira de Engenharia Agrícola e Ambiental, v.15, p.90-95, 2011.

Roman, E.S.; Vargas, L.; Ribeiro, M.C.F.; Luiz, A.R.M. Influência do orvalho e volume de calda de aplicação na eficácia do glyphosate na dessecação de Brachiaria plantaginea. Planta Daninha, v.22, n.3, p.479-482, 2004.
Santos, J.B.; Cury, J.P. Picão-Preto: Uma planta daninha especial em solos tropicais. Planta Daninha, v.29, p.1159-1171, 2011.

Souza, L.A.; Cunha, J.P.A.R; Pavanin, L.A. Deposição do herbicida 2,4-D Amina com diferentes volumes e pontas de pulverização em plantas infestantes. Revista Ciência Agronômica, v.43, n.1, p.78-85, 2012.

Stanislavski, W.M.; Antuniassi, U.R.; Chechetto, R.G. Air flow humidification for airassisted boom sprayer. Engenharia Agrícola, v.34, n.1, p.48-56, 2014.

Stopps, G.J.; Nurse, R.E.; Sikkema, P.H. The effect of time of day on the activity of postemergence soybean herbicides. Weed Technology, v.27, n.4, p.690-695, 2013.

Vilela, C.M.; Antuniassi, U.R. Evaporação de gotas de caldas contendo fungicidas $\mathrm{e}$ adjuvantes depositadas em superfície. Engenharia Agrícola, v.28, n.2, p.65-73, 2013.

Wakabayashi, K.; Böger, P. General physiological characteristics and mode of action of peroxidizing herbicides. SpringerVerlag, Berlin Heidelberg, p.163-190, 1999. 